\title{
Mycoremediation of Naphthenic Acids Sourced from Oil Sands Process Affected Water
}

\author{
Sarah Miles, Ania Ulrich \\ Department of Civil and Environmental Engineering, University of Alberta \\ 9211 - 116 Street NW, Edmonton, Canada \\ smmiles@ualberta.ca; aulrich@ualberta.ca
}

\section{Extended Abstract}

Extraction of bitumen from Alberta's oil sands requires large volumes of water, leading to an abundance of oil sands process-affected water (OSPW) that must be remediated prior to discharge or reuse [1]. OSPW contains a variety of toxic organic compounds, including polyaromatic hydrocarbons (PAH), benzene, toluene, ethylbenzene, xylenes (BTEX), and naphthenic acids (NAs). NAs are known to be recalcitrant contaminants in OSPW, and a significant contributor to toxicity, which poses a major threat to the environment [2]. With the ample presence of organic compounds, microbial communities are highly active in tailings ponds. Previous studies have shown that NAs can be removed from OSPW through microbial degradation under aerobic conditions, and thereby decreasing the toxicity of OSPW [3].

NAs have become the primary target of remedial efforts in tailings treatment undertaken by industrial operators. Ongoing efforts into a cost and energy efficient remediation strategy of NAs have focused on bioremediation. However, limited research has been conducted on the use of mycoremediation in the treatment of NAs. The objective of my research is to characterize the capacity for NAs degradation of the fungal isolate Trichoderma harzianum, and to harness this process for surface OSPW mycoremediation.

Through selective enrichment of OSPW, a microbial community enriched in NA-degraders was established, and several microbial isolates were identified including for the first time a fungal isolate T. harzianum. To effectively characterize the ability of the fungal isolate to degrade NAs, a microcosm study was conducted, containing OSPW-extracted NAs or commercially-produced NAs as the sole source of carbon. NA concentrations were monitored over time to determine degradation capacity and $\mathrm{CO}_{2}$ production was monitored as a proxy of fungal growth. Initial results indicate successful degradation with $23 \%-47 \%$ removal in 126 days of commercially-produced NAs as per GC-FID. Additional microcosms were prepared using model NAs, specifically cyclohexane carboxylic acid (CHCA), and 1-adamantane carboxylic acid (ACA). These model compounds were chosen to determine what possible effect NA cyclicity has on degradation potential. As CHCA is a single ring it potentially is the most easily degraded compound, and ACA with 3 rings is the most complex model NA available and the most difficult to degrade. Initial results indicate successful degradation of ACA with approximately $13 \%$ removal in 126 days as per HPLC-DAD. As ACA is often used as a negative control for biodegradation due to its inability to be biologically degraded. Finding T. harzianum possesses the capacity to degrade ACA is significant indicating perhaps the capacity for degradation of much larger complex ringed NAs.

Alberta's oil sands industry has become heavily scrutinized, both within Canada and worldwide. An increased effort in limiting and rectifying the environmental impact from this industry will contribute to the reestablishment of Canada as a leading voice in environmental stewardship. The findings of this research will contribute to the framework needed to create energy efficient remediation efforts by industrial operators in the Alberta oil sands region, and will assist Canada to limit the environmental impact of the oil sands industry.

\section{References}

[1] Suncor Energy, (2005), Suncor Energy 2005 Report on Sustainability. [Online]. Available: http://www.suncor.com/data/1/rec_docs/616_Suncor\%20SD\%20Report_2005\%20.pdf

[2] C. Whitby, "Microbial naphthenic Acid degradation," Advances in applied microbiology, vol. 70, pp. 93-125, 2010.

[3] L. F. Del Rio, A. K. M.Hadwin, L. J. Pinto, M. D. MacKinnon, and M. M. Moore, "Degradation of naphthenic acids by sediment micro-organisms," Journal of Applied Microbiology, vol. 101, no. 5, pp. 1049-1061, 2006. 